\title{
Heavy Quark Production at CMS and ATLAS
}

\author{
Valery P. Andreev* \\ University of California Los Angeles, \\ Department of Physics and Astronomy, \\ 405 Hilgard Avenue, \\ Los Angeles, CA 90024-1547, USA \\ E-mail: Valeri. Andreevacern.ch
}

\begin{abstract}
By measuring production cross-sections of beauty and charm hadrons and of the heavy-flavour quarkonia, $J / \Psi$ and $Y$, ATLAS and CMS will provide sensitive tests of QCD predictions of production in proton-proton collisions at the LHC. At the LHC new opportunities to improve our understanding of the physics of $b$ quarks will be available because of the high statistics data samples and the high centre-of-mass energy. The capabilities to measure the inclusive and exclusive cross-sections and correlations at the LHC start-up luminosities $\left(\sim 10 \mathrm{pb}^{-1}\right)$ and with the high-luminosity runs $\left(\sim 10 \mathrm{fb}^{-1}\right)$ are presented.
\end{abstract}

12th International Conference on B-Physics at Hadron Machines - BEAUTY 2009

Heidelberg, Germany

September 07-11, 2009

Speaker 


\section{Introduction}

With the start-up of the Large Hadron Collider (LHC), a new energy domain will be explored. This will allow testing production cross-sections of beauty and charm hadrons and of the heavy-flavour quarkonia, $J / \Psi$ and $Y$, measured with the new data against the framework of quantum chromodynamics (QCD).

Two general-purpose detectors, ATLAS [1] and CMS [2], designed to explore the physics at the $\mathrm{TeV}$ scale, have an extensive research program for heavy-flavour studies. The two experiments are very different in the layout chosen for the magnet system. In ATLAS, a solenoid provides the magnetic field for the Inner Tracker, while a system of air-core toroids outside the calorimeters provides the field for the Muon Spectrometer. The magnetic field in CMS is provided by a single very large solenoid which contains both the Inner Tracker and the calorimeters. The muon chambers are embedded in the iron of the solenoid return yoke. The magnet layout determines the weight (ATLAS is larger but lighter) of the two detectors. The potential of ATLAS and CMS for heavy-flavour measurements is presented for the LHC startup luminosities $\left(10 \mathrm{pb}^{-1}\right.$ or so) and for the high-luminosity runs $\left(10 \mathrm{fb}^{-1}\right)$.

Details of the triggers to be used in $B$ (heavy-flavour) physics programme by ATLAS and CMS can be found in [3] and [4], respectively.

\section{Quarkonia production}

\subsection{Quarkonia production cross section.}

The prompt production of quarkonia continues to be puzzling. There is a variety of production models available for prompt quarkonium production [5], among which are the Colour Singlet Model (CSM) and the Colour Octet Model (COM).

Beauty and quarkonia studies will play an important role in the early data-taking at low luminosity $\left(10^{31} \mathrm{~cm}^{-2} \mathrm{~s}^{-1}\right)$. A calibration method to obtain the low- $p_{T}$ muon trigger efficiency and the muon reconstruction efficiency using real data by virtue of the so-called tag-and-probe method with quarkonia reconstruction is developed [3,4]. With an integrated luminosity of 10 $\mathrm{pb}^{-1}$ ATLAS and CMS will be able to register about $\sim 10^{5}$ events containing $\mathrm{J} / \psi \rightarrow \mu^{+} \mu^{-}$ selected by the low luminosity trigger menu. Reconstructed events will contain $\mathrm{J} / \psi \rightarrow \mu^{+} \mu^{-}$ produced both directly in proton-proton interactions as well as indirectly from decays of $\mathrm{B}$ hadrons.

The dimuon triggers dedicated to quarkonium are: the topological dimuon trigger (ATLAS), corresponding to two muon candidates with $p_{T}$ thresholds of 6 and $4 \mathrm{GeV}(\mu 6 \mu 4$ trigger) and High Level Trigger (CMS) with $p_{T}$ threshold of $3 \mathrm{GeV}$ for both muons. An additional trigger scenario is based on a single muon trigger with a higher $p_{T}$ threshold. Figure 1 shows the quarkonium signal and main background invariant mass distributions in ATLAS for those events which passed the trigger requirements, with reconstruction efficiencies and background suppression cuts taken into account. Peaks from the $J / \psi$ and $Y(1 \mathrm{~S})$ clearly dominate the background. 


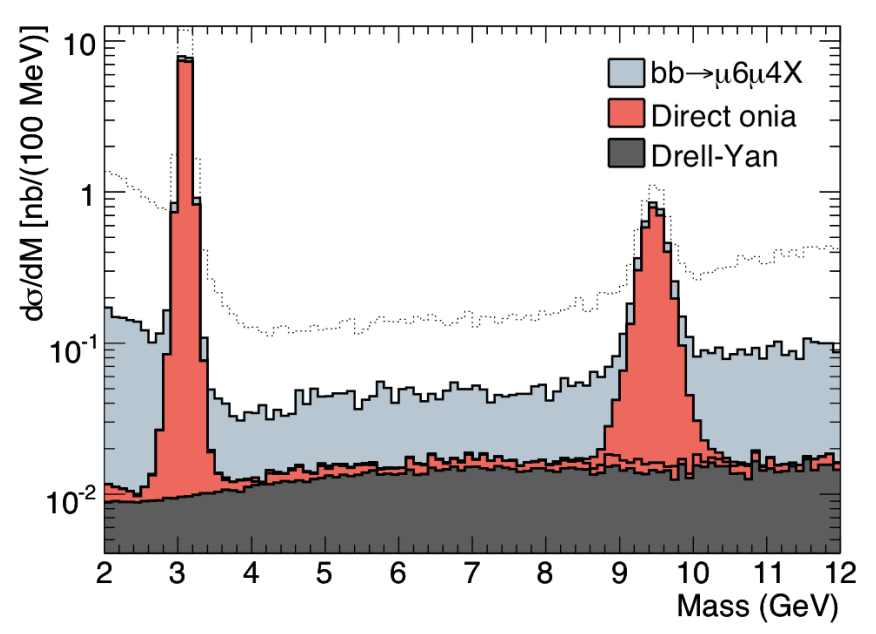

Figure 1. ATLAS quarkonia selection. The cumulative plot of the invariant mass of dimuons from various sources, reconstructed with a $\mu 6 \mu 4$ trigger, with the requirement that both muons are identified as coming from the primary vertex and with a pseudo-proper time cut of $0.2 \mathrm{ps}$. The dotted line shows the cumulative distribution without vertex and pseudo-proper time cuts.

The precision tracking of ATLAS and CMS permits to disentangle the prompt $\mathrm{J} / \psi$ production from $\mathrm{B}$ hadron decays, and therefore allows to determine the $\mathrm{B}$ hadron cross section $[3,6]$. Contributions from prompt and non-prompt $\mathrm{J} / \psi$ 's are separated by using lifetime distributions. Figure 2 displays the prompt J/ $\psi$ differential cross sections in CMS [6], with combined systematic and statistical uncertainties, corresponding to an integrated luminosity of $3 \mathrm{pb}^{-1}$. The precision of the result is limited by systematic uncertainties, and is at the $15 \%$ level. Fig. 3 shows the result of the Monte Carlo (MC) fits to the fraction of J/\%'s from B hadron decay in each bin of $\mathrm{p}_{\mathrm{T}}^{\mathrm{J} / \psi}$.

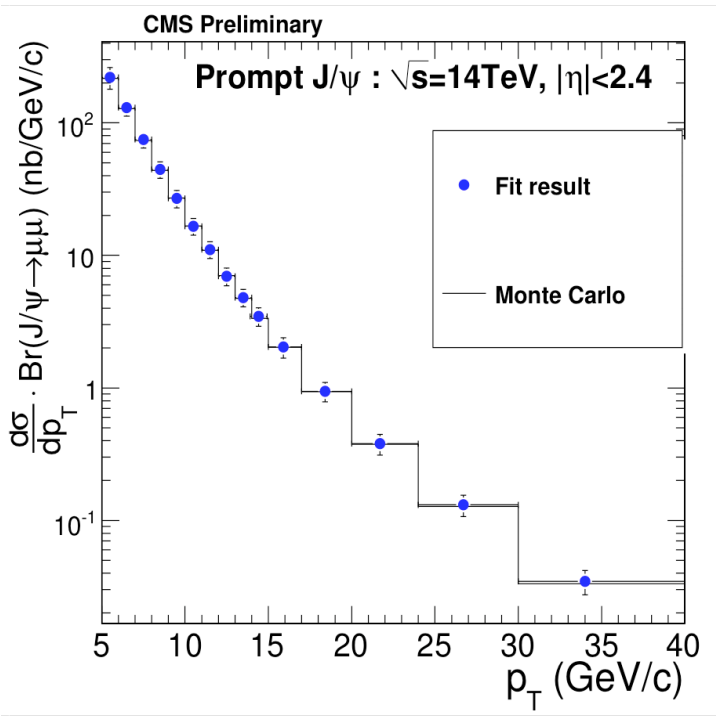

Figure 2. The expected prompt $\mathrm{J} / \psi$ differential cross section obtained from a sample of Monte Carlo simulated events corresponding to $3 \mathrm{pb}^{-1}$ of data collected by the CMS detector. The cross section is plotted as a function of the $\mathrm{J} / \psi \mathrm{p}_{\mathrm{T}}$, integrated over the pseudorapidity range $\left|\eta^{\mathrm{J} / \psi}\right|<2.4$. 


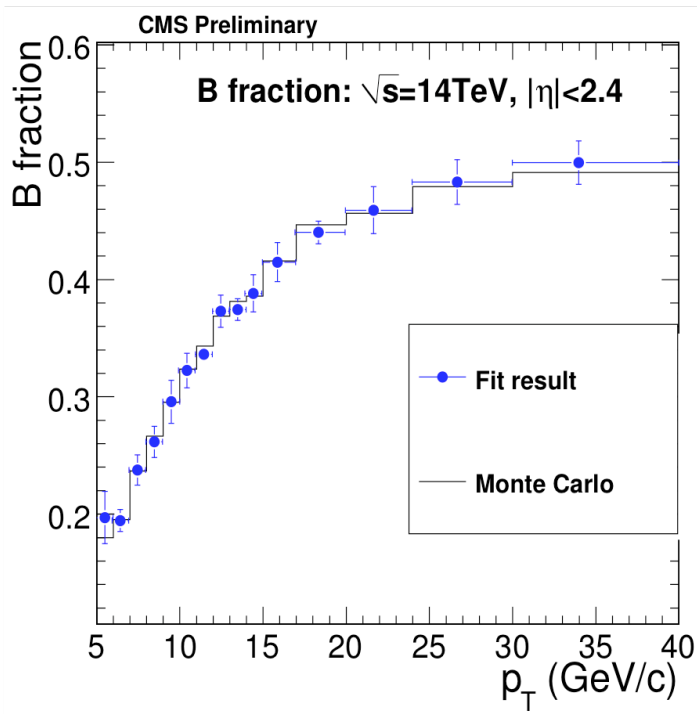

Figure 3. The fitted fraction of $\mathrm{J} / \psi$ from $B$ hadron decays obtained from a sample of Monte Carlo simulated events corresponding to $3 \mathrm{pb}^{-1}$ of data collected by the CMS detector. The fitted fraction of $\mathrm{J} / \psi$ is plotted as a function of $\mathrm{J} / \psi \mathrm{p}_{\mathrm{T}}$, integrated over the pseudorapidity range $\left|\eta^{J / \psi}\right|<2.4$.

\subsection{Quarkonia polarization.}

The measurement of the quarkonia production cross section is the first step to investigate the mechanism of the quarkonium hadroproduction. With more data it will be possible to measure the prompt $J / \Psi$ and $Y$ polarizations. Quarkonium polarization will be measured by analyzing the angular distribution of the muons produced in the quarkonium decay. The polarization measurement is important for discrimination of the production models. The Colour Octet Model predicts that the prompt quarkonia produced in $p p$ collisions are transversely polarized, with the polarization increasing as a function of the transverse momentum. Other production models predict different $p_{T}$ dependencies of the polarization. ATLAS expectations on $J / \Psi$ and $Y$ polarization and cross section measurement with $10 \mathrm{pb}^{-1}$ [3], are presented in Table 1.

Table 1. ATLAS prospects for $J / \Psi$ and $Y$ polarization and cross-sections measurements in slices of $p_{T}$, for $10 \mathrm{pb}^{-1}$.

\begin{tabular}{|c||c||r|r|r|r|r|r|}
\hline Sample & $p_{T}, \mathrm{GeV}$ & $9-12$ & $12-13$ & $13-15$ & $15-17$ & $17-21$ & $>21$ \\
\hline \hline \multirow{5}{*}{$J / \psi, \alpha_{\text {gen }}=0$} & $\alpha$ & 0.156 & -0.006 & 0.004 & -0.003 & -0.039 & 0.019 \\
& & \pm 0.166 & \pm 0.032 & \pm 0.029 & \pm 0.037 & \pm 0.038 & \pm 0.057 \\
\cline { 2 - 8 } & $\sigma, \mathrm{nb}$ & 87.45 & 9.85 & 11.02 & 5.29 & 4.15 & 2.52 \\
& & \pm 4.35 & \pm 0.09 & \pm 0.09 & \pm 0.05 & \pm 0.04 & \pm 0.04 \\
\hline \hline \multirow{5}{*}{$J / \psi, \alpha_{\text {gen }}=+1$} & $\alpha$ & 1.268 & 0.998 & 1.008 & 0.9964 & 0.9320 & 1.0217 \\
& & \pm 0.290 & \pm 0.049 & \pm 0.044 & \pm 0.054 & \pm 0.056 & \pm 0.088 \\
\cline { 2 - 8 } & $\sigma, \mathrm{nb}$ & 117.96 & 13.14 & 14.71 & 7.06 & 5.52 & 3.36 \\
& & \pm 6.51 & \pm 0.12 & \pm 0.12 & \pm 0.07 & \pm 0.05 & \pm 0.05 \\
\hline \hline \multirow{5}{*}{$J / \psi, \alpha_{\text {gen }}=-1$} & $\alpha$ & -0.978 & -1.003 & -1.000 & -1.001 & -1.007 & -0.996 \\
& & \pm 0.027 & \pm 0.010 & \pm 0.010 & \pm 0.013 & \pm 0.014 & \pm 0.018 \\
\cline { 2 - 8 } & & 56.74 & 6.58 & 7.34 & 3.53 & 2.78 & 1.68 \\
& & \pm 2.58 & \pm 0.06 & \pm 0.06 & \pm 0.04 & \pm 0.03 & \pm 0.02 \\
\hline \hline \multirow{3}{*}{$\alpha_{\text {gen }}=0$} & $\alpha$ & -0.42 & -0.38 & -0.20 & 0.08 & -0.15 & 0.47 \\
& & \pm 0.17 & \pm 0.22 & \pm 0.20 & \pm 0.22 & \pm 0.18 & \pm 0.22 \\
\cline { 2 - 7 } & $\sigma, \mathrm{nb}$ & 2.523 & 0.444 & 0.584 & 0.330 & 0.329 & 0.284 \\
& & \pm 0.127 & \pm 0.027 & \pm 0.029 & \pm 0.016 & \pm 0.015 & \pm 0.012 \\
\hline
\end{tabular}

With the integrated luminosity of $10 \mathrm{pb}^{-1}$ it will be possible to measure the polarization of $J / \psi$ with the precision of order $0.02-0.06$ in a wide range of transverse momenta. In case of $Y$, the expected precision is of order 0.20 . 


\section{Beauty production}

\subsection{Exclusive B production.}

Further on with luminosity increase the exclusive decays of $\mathrm{B}^{+} \rightarrow \mathrm{J} / \psi \mathrm{K}^{+}, \mathrm{B} 0 \rightarrow \mathrm{J} / \psi \mathrm{K}^{*} 0$ and $\mathrm{B}^{0} \rightarrow \mathrm{J} / \psi \varphi$ will be studied. These channels can be observed at LHC with the first $10 \mathrm{pb}^{-1}$ of data and can be used for detector performance studies. The exclusive cross section measurements have different systematic uncertainties and model dependencies (fragmentation) from the inclusive ones. The $b$ production represents the largest physics background for many new physics searches, therefore its measurement is a prerequisite to any discovery. The feasibility study of measuring with early CMS data the differential production cross section $\mathrm{d} \sigma / \mathrm{dp}_{\mathrm{T}}(\mathrm{B})$ and lifetime ratio $\tau\left(\mathrm{B}^{+}\right) / \tau\left(\mathrm{B}^{0}\right)$ using fully reconstructed $\mathrm{B}^{+} \rightarrow \mathrm{J} / \psi \mathrm{K}^{+}$and $\mathrm{B}^{0} \rightarrow \mathrm{J} / \psi \mathrm{K}^{* 0}$ decays is presented in [7]. The $J / \psi$ and $\mathrm{K}^{* 0}$ are reconstructed in the $\mu^{+} \mu^{-}$and $\mathrm{K}^{+} \pi^{-}$channels, respectively. The lifetime in each decay channel is determined using an unbinned maximum likelihood fit to the invariant mass and proper decay length of the reconstructed B candidates. The fit result with individual contributions from the various components is illustrated in Fig. 4.
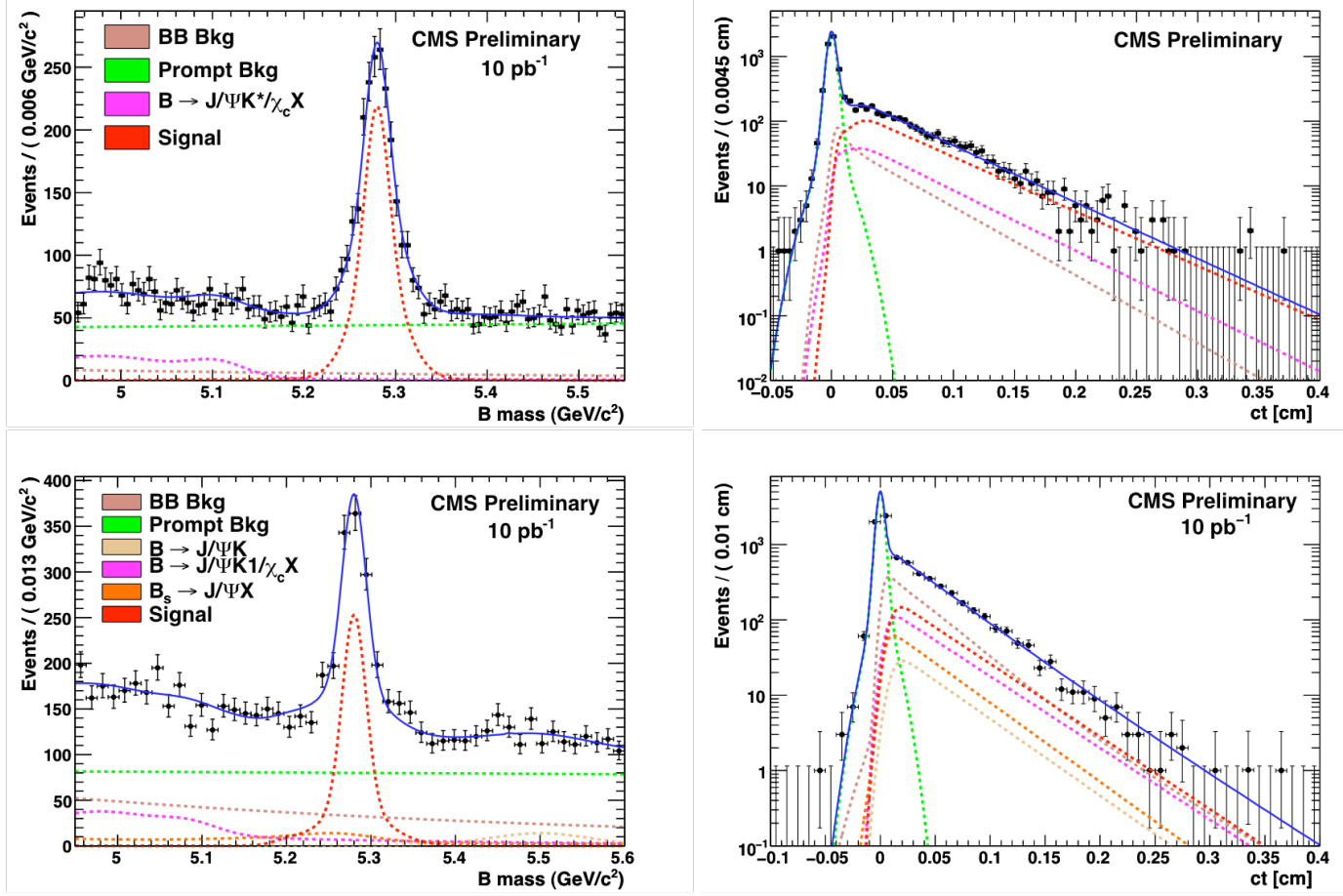

Figure 4. CMS distributions of $M_{\mathrm{B}}$ and $c t$ for the $\mathrm{B}^{+}$(top) and $\mathrm{B}^{0}$ (bottom) fits integrating over all $p_{T}$ bins. Individual contributions from the various components are shown in different colors (refer to legend in the plots).

Feasibility study demonstrated that the differential cross sections and lifetime ratio for the exclusive decays $\mathrm{B}^{+} \rightarrow \mathrm{J} / \psi \mathrm{K}^{+}$and $\mathrm{B}^{0} \rightarrow \mathrm{J} / \psi \mathrm{K}^{* 0}$ can be measured to a statistical precision of less than $10 \%$ on the cross section and $5 \%$ on the lifetime ratio using as little as $10 \mathrm{pb}^{-1}$ of calibrated data. The measurement of the cross section is dominated by systematics, for which the largest contribution is expected to come from the luminosity uncertainty. The lifetime ratio 
is dominated by the statistical uncertainty. Similar expected precision for the cross section measurement in the exclusive decay $\mathrm{B}^{+} \rightarrow \mathrm{J} / \psi \mathrm{K}^{+}$has been obtained in the ATLAS study [3].

\subsection{Correlations.}

Three mechanisms contribute to the beauty production at hadron colliders: gluon-gluon fusion and $q \bar{q}$ annihilation (flavour creation in hard QCD scattering), flavour excitation (semihard process) and gluon splitting (soft process). Flavour creation refers to the lowest-order, two-to-two QCD $b \bar{b}$ production diagrams. Flavour excitation corresponds to diagrams where a $b \bar{b}$ pair from the quark sea of the proton is excited into the final state due to one of the $b$ quarks undergoes a hard QCD interaction with a parton from the other proton. Gluon splitting refers to the processes in which the $b \bar{b}$ pair arises from a $g \rightarrow b \bar{b}$ splitting in the initial or final state. Neither of the quarks from $b \bar{b}$ pair participate in the hard QCD scattering in this case.

Correlation measurements are foreseen at LHC in order to study details of the production mechanisms discussed above. The angular distance $\Delta \phi$ between the $b$ quark directions in the transverse plane is the main discriminating variable to disentangle contributions from the gluon-gluon fusion, gluon splitting and flavour excitation. The $\Delta \phi$ distribution for gluon splitting is slightly peaked at small $\Delta \phi$ values. The angle between the two $b$-quarks produced by the gluon-fusion mechanism has a peak at 180 degrees, as expected, since in the process $g g$ $\rightarrow b \bar{b}$ the $b$ quarks are produced back-to-back in the transverse plane. For the flavour excitation production mechanism the back-to-back topology is preferred too.

The MC study with the CMS detector simulation using a clean fully leptonic signature is presented in [8]. The decay of one $b$ quark is tagged by reconstructing the decay $\mathrm{J} / \psi \rightarrow \mu^{+} \mu^{-}$. Events are also required to contain an additional muon consistent with the semileptonic decay of the second $b$ quark. The angular distance $\Delta \phi$ between the $\mathrm{J} / \psi$ and muon after all the selection criteria is shown in Fig. 5 (left).
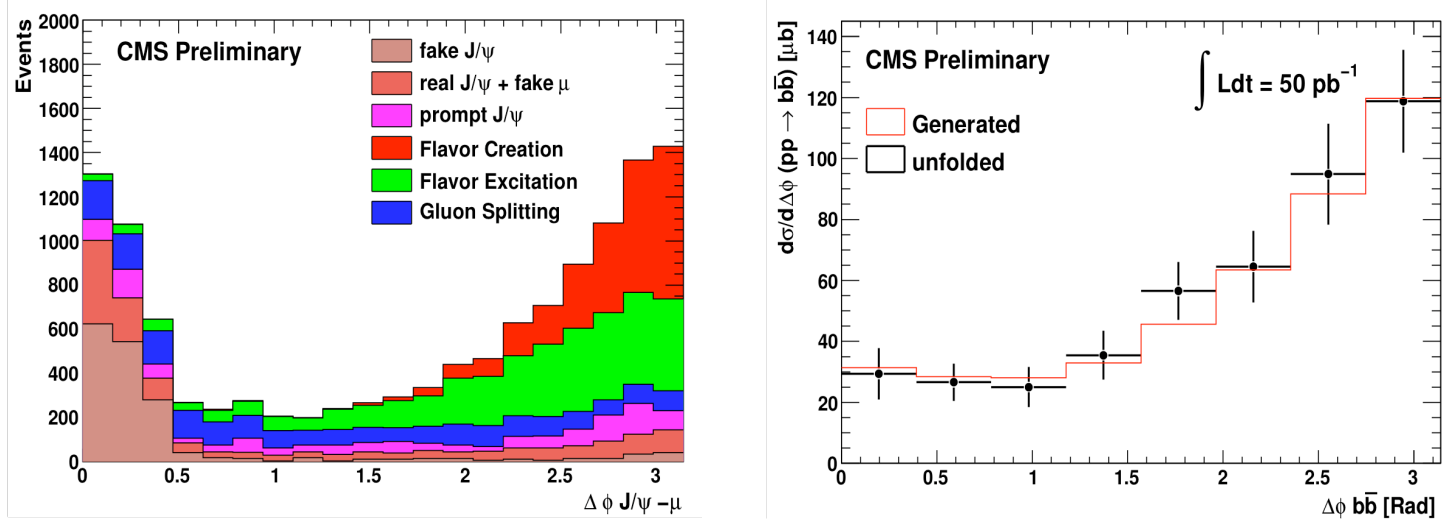

Figure 5. Left: $\mathrm{J} / \psi-\mu \Delta \phi$ distribution after all the selection criteria. The different truth-matched components are stacked and shown in different colors. Right: differential cross section measurement $\mathrm{d} \sigma / \mathrm{d} \Delta \phi$ after unfolding and including systematic uncertainties.

Fig. 5 (right) shows the differential cross section measurement as determined on a Monte Carlo sample corresponding to $50 \mathrm{pb}^{-1}$. The unfolding procedure has been applied to correct for a significant migration of events generated in one bin of $\Delta \phi(b \bar{b})$ and ending up in a 
different bin of reconstructed $\Delta \varphi$ between the $\mathrm{J} / \psi$ and muon candidates. Depending on the particular $\Delta \phi$ bin, an accuracy of $15-25 \%$ on the differential cross-section can be obtained, combining statistical and systematic uncertainty. An accuracy at the $10 \%$ level is expected for the integrated total cross section.

\subsection{Inclusive B production.}

A study [9] has been performed in CMS to investigate methods of identifying of b-jets ( $b$ "tagging") in an inclusive sample of events containing jets and at least one muon for a high statistics event sample $\left(10 \mathrm{fb}^{-1}\right)$ at $14 \mathrm{TeV}$ center-of-mass energy. The CMS capability to measure the inclusive $b$-quark production cross section as a function of the $\mathrm{B}$ hadron transverse momentum $p_{\mathrm{T}}$ and pseudorapidity $\eta$ is presented here. The measurement of the differential cross sections is studied for B hadrons of $p_{\mathrm{T}}>50 \mathrm{GeV} / \mathrm{c}$ and within the fiducial volume of $|\eta|<2.4$. The event selection requires a b-tagged jet in the fiducial volume to be present in the event. $B$ tagging is based on inclusive secondary-vertex reconstruction in jets. At Level-1 trigger, the single-muon trigger is used. At the High Level Trigger the "muon + b-jet" trigger is required. The most energetic $B$ hadron inside the phase space defined above is selected. Good correspondence between the generated B particle and the most energetic reconstructed b-tagged jet is observed. The corresponding relative resolutions for B particles with $p_{\mathrm{T}}>170 \mathrm{GeV} / \mathrm{c}$ are $13 \%$ and $6 \%$ for $p_{\mathrm{T}}$ and pseudorapidity, respectively. The average btagging efficiency is $65 \%$ in the barrel region, while the efficiency is about $10 \%$ less for the endcap region. The signal fraction is determined from a fit to the data distribution using the simulated shapes for the signal and background. To do so, a lepton tag by selecting inclusive muons is applied. Each reconstructed muon is associated to the most energetic b-tagged jet. The muon must be closer to this b-tagged jet than to any other jet in the event. In most cases the tagged muon is inside the b-jet. The average efficiency of associating the muon with the btagged jet is $75 \%$. The transverse momentum of the muon with respect to the b-jet axis effectively discriminates between $b$ events and background.

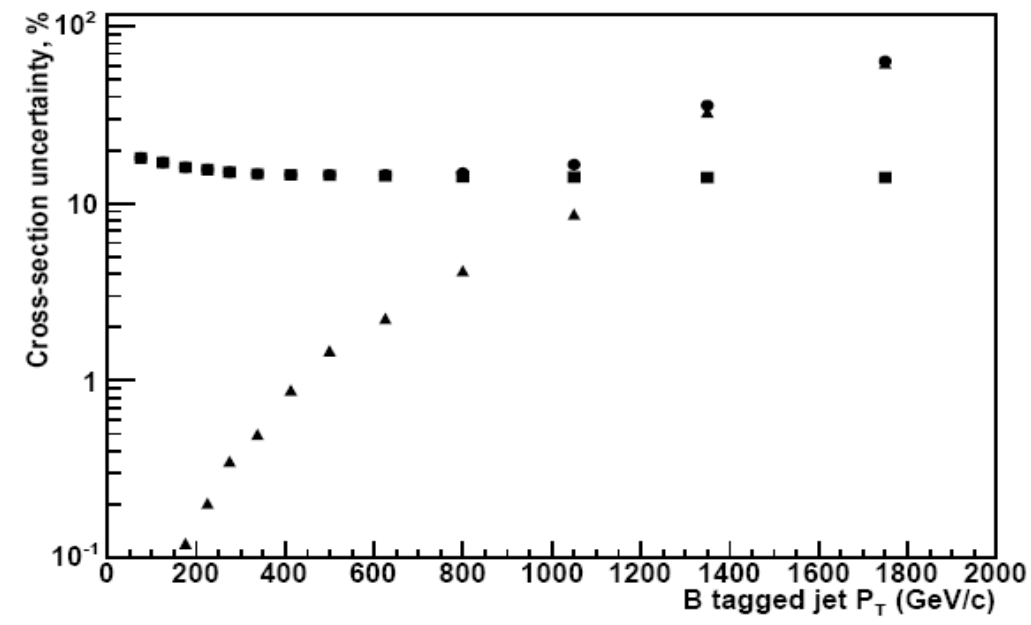

Figure 6. The statistical uncertainty for the CMS inclusive $b$ cross section measurement (triangles), systematic (squares) uncertainty and total (dots) uncertainty as function of the b-tagged jet transverse momentum with respect to the beam line. Total uncertainty comprises the statistical and systematic uncertainties added in quadrature. 
Several potential sources of systematic uncertainties are considered and their impact on the observed cross section is detailed in [9]. The largest uncertainty arises from the $3 \%$ error on the jet energy scale which leads to a cross section error of $12 \%$ at $E_{\mathrm{T}}>50 \mathrm{GeV} / \mathrm{c}$. The estimated statistical, systematic and total uncertainty as function of the b-tagged jet transverse momentum with respect to the beam line is shown in Fig. 6.

The event selection for inclusive $b$ production measurement at CMS will allow to study $b$ production mechanisms on an event sample of 16 million $b$ events for $10 \mathrm{fb}^{-1}$ of integrated luminosity. The $b$ purity of the selected events varies as function of the transverse momentum in a range from $70 \%$ to $55 \%$. The estimate shows that with the CMS detector one can reach $1.5 \mathrm{TeV} / \mathrm{c}$ as the highest measured transverse momentum of $\mathrm{B}$ hadrons. The results are preliminary, the improvements are likely as further jet calibration tunings, software and analysis algorithm developments are foreseen.

\section{Acknowledgements}

We thank Paula Eerola and Jim Olsen (CMS B physics group conveners) and Samira Hassani and Chara Petridou (ATLAS B physics group conveners) for their help with the talk preparation.

\section{References}

[1] ATLAS Collaboration, The ATLAS experiment at the CERN LHC, [JINST 3 (2008) S08003].

[2] CMS Collaboration, The CMS experiment at the CERN LHC, [JINST 3 (2008) S08004].

[3] ATLAS Collaboration, Expected performance of the ATLAS experiment: detector, trigger and physics [CERN-OPEN-2008-20, 2009].

[4] CMS Collaboration, The Trigger and Data Acquisition Project, Vol. II [CERN/LHCC 2002-026, 2002].

[5] N. Brambilla et al. CERN Yellow Report, [CERN-2005-005, 2005].

[6] CMS Collaboration. Feasibility study of a J/\% cross section measurement with early CMS data. [CMS PAS BPH-07-002, 2008].

[7] CMS Collaboration. Measurement of Differential Production Cross Sections and Lifetime Ratio for Exclusive Decays of $B^{+}$and $B^{0}$ Mesons in pp Collisions at $\sqrt{s}_{s}=10 \mathrm{TeV}$ [CMS PAS BPH-09001, 2009].

[8] CMS Collaboration. Measurement of the Azimuthal Correlation in b $\bar{b}$ Production in $p p$ Collisions with the CMS detector [CMS PAS BPH-08-004, 2009].

[9] V. P. Andreev, D. B. Cline and S. Otwinowski. Measurement of open beauty production at LHC with CMS. [CMS Note 2006/120, 2006]. 